\title{
Prurigogestationis leading to prurigonodularis resulting in permanent disfiguration of the skin
}

\author{
N Senewiratne ${ }^{1}$, N Karunarathne ${ }^{1}$, S Nanayakkara ${ }^{1}$, G R C Silva ${ }^{1}$, Achala W Abeywardena ${ }^{1}$, Prabath K \\ Abeysundara ${ }^{1}$
}

Sri Lanka Journal of Obstetrics and Gynaecology 2012; 34: 51-52

\begin{abstract}
Dermatoses of pregnancy are common, but rarely affect the feto-maternal outcome. Prurigogestationis is a pregnancy specific dermatological condition, which resolves spontaneously in the puerperium. We report a rare case in which it led to permanent disfiguration of the skin by transformation in to prurigonodularis.
\end{abstract}

Key words: dermatoses of pregnancy, prurigonodularis

\section{Introduction}

Prurigogestationis is a common skin condition in pregnancy, which is characterized by small, erythematous or skin-coloured papules that are extremely pruritic. Excoriated papules are seen on the proximal limbs and upper trunk, most often occurring between the 20th and 34th week of gestation. It has an estimated prevalence of one in 300 pregnancies. Besnierprurigo, early-onset prurigo of pregnancy, linear IgM dermatosis of pregnancy, papular dermatitis of pregnancy, Spangler's papular dermatitis of pregnancy are different terms which denote prurigogestationis. The exact etiology of this condition is unknown, but it is considered likely to be a flare-up of atopic dermatitis during pregnancy.

Prurigonodularis was first described by Hyde and Montgomery in 1909. It is a dermatological condition which is classified in ICD-10, under dermatitis and eczema. The exact etiology of nodular prurigo is unknown. But It is associated with Becker's nevus, linear IgA disease, liver disease, T cell diseases, cholestasis, thyroid diseases, polycythaemia-

\footnotetext{
${ }^{1}$ De Soysa Hospital for Women, Colombo 08, Sri Lanka. Correspondence: Prabath K Abeysundara E-mail: heshanprabathkularathne@yahoo.com
}

rubravera, uraemia, Hodgkin's disease, HIV, internal malignancies, liver failure, renal failure, psychiatric illnesses and immunodeficiency diseases.

We report a rare case where prurigogestationis led to prurigonodularis which resulted in scarring of the skin.

\section{Case report}

A 23-year old mother in her first pregnancy, who has a past history of childhood atopic eczema presented at 20 weeks of period of gestation with severe pruritus. Small erythematous papules appeared on the extensor surfaces of both upper and lower limbs. Propagation of the skin lesions was distal to proximal. Face, trunk, both palms and soles were spared. She was afebrile and anicteric. Serum alkaline phosphatase, bilirubin, liver enzymes, bile acids and inflammatory markers were normal. Regular fetal growth scans revealed a normal growth of the fetus. The clinical picture of the condition was compatible with prurigogestationis.

Pruritus appeared extensive towards the end of the pregnancy and did not respond to antihistamines. Papules transformed in to skin nodules of various sizes. Nodules became discrete, hyper pigmented and firm. They were excoriated lesions with crusted tops. Size of the nodules varied between one to three centimetres in its largest diameter. But there were no features of acute inflammation (Figure 1).

Scrapings of the lesions were sent for microbiological assessment which did not reveal any bacterial growth. Histopathological assessment revealed irregular acanthosis associated with hyperkeratosis and focal parakeratosis in the epidermis. Dermis showed increased number of small blood vessels, fibrosis with vertical collagen bundles in the papillary dermis. Mild inflammatory cell infiltrate was also noted. Findings were compatible with prurigonodularis (Figure 2). 


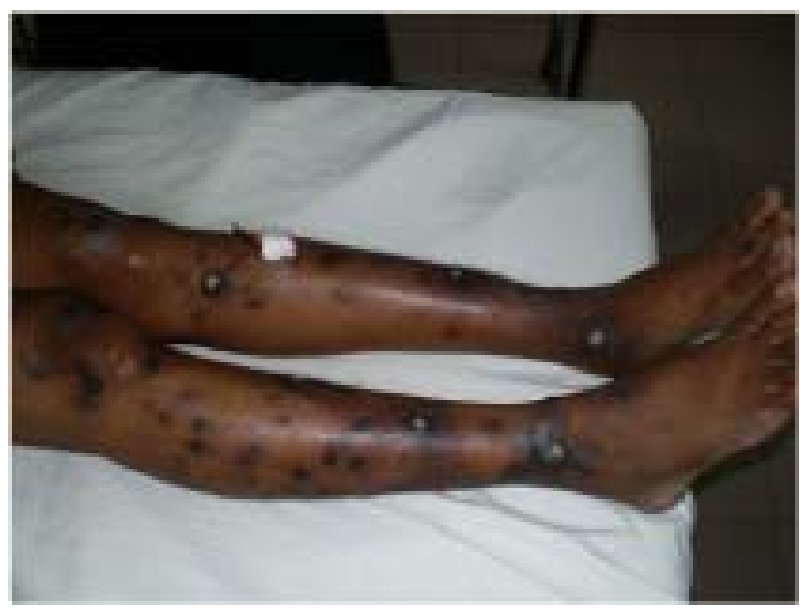

Figure 1. Excoriated nodules of prurigonodularis.

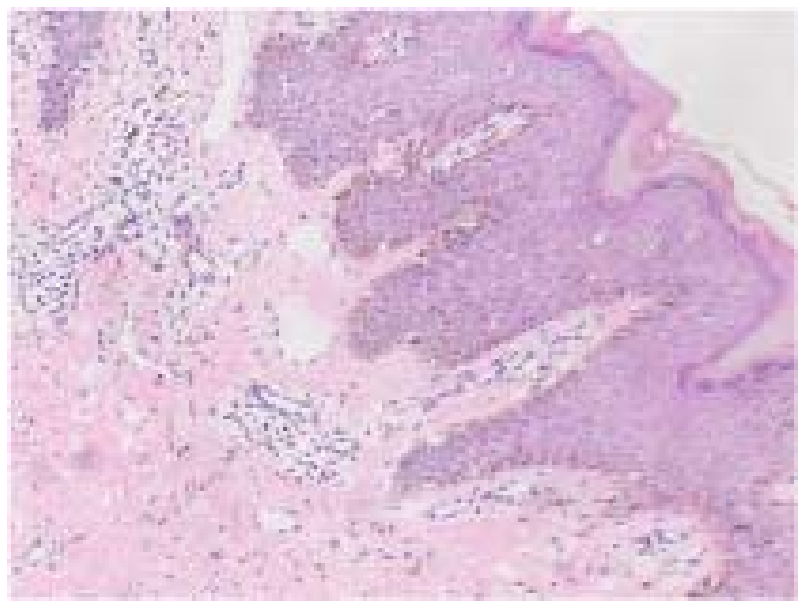

Figure 2. Histological appearance of prurigonodularis; haematoxylin and eosin stain.

$(20 \times 10$ magnification)

Pregnancy ended up with a vaginal delivery at term which delivered a healthy baby boy weighing $2.8 \mathrm{~kg}$. Following the delivery pruritus disappeared but the skin lesions persisted with permanent disfiguration of the skin. Patient was referred to the dermatological clinic and there she was treated with intra lesion steroids.

\section{Discussion}

The skin changes in pregnancy can be either physiological, changes in pre-existing skin diseases or development of new pregnancy specific dermatoses. Pregnancy-specific skin conditions include an ill- defined heterogeneous group of pruritic skin eruptions which are seen only in pregnancy. These include atopic eruption of pregnancy, polymorphic eruption of pregnancy, prurigogestationis and intrahepatic cholestasis of pregnancy. Antepartum surveillance is recommended for patients with pemphigoid gestationis and intrahepatic cholestasis of pregnancy as they carry fetal risk. Most skin eruptions including prurigogestationis resolve postpartum and require only symptomatic treatment.

But in our patient, papules of prurigogestationis transmuted in to nodules and did not resolve spontaneously. Permanent disfiguration of the skin followed due to scarring of the excoriated skin nodules. History of childhood atopic eczema would have been a predisposing factor for the transformation of prurigogestationis to prurigonodularis. The association between childhood atopic eczema and transformation of prurigogestationis in to prurigonodularis needs further evaluation.

\section{Acknowledgements}

Dr. J. T. S. Maduranga, Intern House Officer of De Soysa Hospital for Women, Colombo 08, is acknowledged for the provision of the digital images of the skin lesions.

\section{References}

1. Wen FAN, Yun QU. Images for diagnosis: prurigogestationis. Chin Med J 2010; 123: 638-40.

2. Tunzi M, Gray GR. Common skin conditions during pregnancy. Am Fam Physician 2007; 75: 211-8.

3. Kroumpouzos G, Cohen LM. Specific dermatoses of pregnancy: an evidence-based systematic review. Am JObstet Gynecol 2003; 188: 1083-92.

4. Hyde JN, Montgomery FH. A practical treatise on disease of the skin for the use of students and practitioners. 1909; 174-5.

5. Lockshin BN, Brogan B, Billings S, Billings S. Eczematous dermatitis and prurigonodularis confined to a Becker's nevus. Int J Dermatol 2006; 45: 1465-66.

6. Torchia D, Caproni M, Del Bianco E, Cozzani E, Ketabchi $\mathrm{S}$, Fabbri P. Linear IgA disease presenting as prurigonodularis. Br J Dermatol 2006; 155: 479-80.

7. Hazin R, Abu-Rajab Tamimi TI, Abuzetun JY, Zein NN. Recognizing and treating cutaneous signs of liver disease. Cleve Clin J Med 2009; 76: 599-606. 